\title{
Sulforaphane Inhibits Proliferation and Apoptosis of Colorectal Cancer Cells by Down-Regulating the Cyclooxygenase-2/Protein Kinase B/Glycogen Synthase Kinase-3 Beta Signaling Pathway
}

\author{
Y. ZHU, HAIJUN HU, K. HUANG, XIAO SUN AND MINGWEI LI* \\ Department of Gastrointestinal Surgery, Shenzhen People's Hospital, Luohu, Shenzhen 518020, China
}

Zhu et al.: Sulforaphane Inhibits Proliferation and Apoptosis of Colorectal Cancer Cells

\begin{abstract}
To determine the effects of sulforaphane on the proliferation and apoptosis of colorectal cancer cell line HCT116 and the levels of proteins related to the cyclooxygenase-2/protein kinase B/glycogen synthase kinase-3 beta signal transduction. Colorectal cancer HCT116 cell lines were cultured in vitro and assigned into sulforaphane (30, 60 and $120 \mu \mathrm{M})$ groups, simple cisplatin group and cisplatin+sulforaphane group. The relative survival rate and apoptosis rate of HCT116 cells were determined by cell counting kit-8 assay and flow cytometry, respectively. Besides, the expression levels of proteins survivin, caspase-3, cyclooxygenase-2, protein kinase B, phosphorylated-protein kinase B, glycogen synthase kinase-3 beta and phosphorylated-glycogen synthase kinase- 3 beta in HCT116 cells were measured through Western blotting. Compared with that in control group, the relative survival rate of cells in 60 and $120 \mu \mathrm{M}$ sulforaphane groups, simple cisplatin group and cisplatin+sulforaphane group declined significantly $(p<0.05)$. Sulforaphane can exert its anti-tumor effect by inducing apoptosis and suppressing proliferation of HCT116 cells and its mechanism may be associated with inhibition on the activation of the cyclooxygenase-2/protein kinase B/glycogen synthase kinase-3 beta signaling pathway.
\end{abstract}

Key words: Sulforaphane, colorectal cancer, cyclooxygenase-2, protein kinase B, glycogen synthase kinase-3

Sulforaphane, also known as "raphanin", is an isothiocyanate derivative extensively existing in broccoli, cauliflower, cabbage, radish, mustard and other cruciferous plants ${ }^{[1]}$. Pharmacological studies have revealed that sulforaphane has anti-tumor, antioxidant, anti-inflammatory and immune regulation effects, showing good pharmacological activities in gastric cancer, colon cancer and lung cancer ${ }^{[2,3]}$. According to a study, the occurrence and development of tumor are closely associated with the high expression of Cyclooxygenase-2 (COX-2) and the expression of COX-2 is significantly increased in colorectal cancer cells ${ }^{[4]}$. Early studies have also suggested that highly expressed COX-2 can also induce the Protein Kinase B (Akt)/Glycogen Synthase Kinase-3 beta (GSK3 $\beta$ ) signal transduction and this signal transduction is also involved in biological processes such as proliferation, invasion and migration of colorectal cancer cells $s^{[5,6]}$. However, there have been few studies on whether

*Address for correspondence

E-mail: zhuyongjun850850@163.com

January-February 2022 sulforaphane inhibits the proliferation of colorectal cancer SW480 cells by regulating the activation of the COX-2/Akt/GSK3 $\beta$ signaling pathway. Hence, this study aimed to observe the effects of sulforaphane on the proliferation and apoptosis of colorectal cancer SW480 cells and on the COX-2/Akt/GSK3 $\beta$ signaling pathway. Human colorectal cancer SW480 cell lines were purchased from Wuhan Buffalo Biological Co., Ltd., and Dulbecco's Modified Eagle Medium (DMEM), Fetal Bovine Serum (FBS) and trypsin were sourced from Gibco. Cell Counting Kit-8 (CCK-8) was bought from Solarbio, Radioimmunoprecipitation Assay (RIPA) lysate was provided by Zhejiang Tianhang This is an open access article distributed under the terms of the Creative
Commons Attribution-NonCommercial-ShareAlike 3.0 License, which
allows others to remix, tweak, and build upon the work non-commercially,
as long as the author is credited and the new creations are licensed under
the identical terms

Accepted 03 July 2022

Revised 21 May 2021

Received 02 July 2019

Indian J Pharm Sci 2022;84(1):219-223 
Biotechnology Co., Ltd., and Bicinchoninic Acid (BCA) protein assay kit and Annexin V-Fluorescein Isothiocyante (FITC) apoptosis detection kit were purchased from Beijing 4A Biotech Co., Ltd. In addition, rabbit anti-mouse survivin, caspase-3, COX2 , Akt, phosphorylated (p)-Akt, GSK3 $\beta$, p-GSK3 $\beta$ and $\beta$-actin antibodies were sourced from CST (USA), goat anti-rabbit Immunoglobulin $\mathrm{G}$ (IgG) secondary antibody was provided by Shanghai Guge Biological TechnologyCo.,Ltd., andEnhancedChemiluminescence (ECL) assay kit was bought from Advansta. Bio-Rad 550 microplate reader (BIO-RAD, USA), Amersham Imager 600 (GE Healthcare, USA) and FACSCalibur flow cytometer (Becton, Dickinson and Company, USA) were prepared. SW480 cells were cultured in DMEM containing $10 \%$ FBS and $1 \%$ antibiotics under $5 \%$ carbon dioxide at $37^{\circ}$. When the cells reached about $90 \%$ confluence, they were subcultured, specifically as follows: The cells were washed with Phosphate-Buffered Saline (PBS) twice, digested with trypsin and then added with culture medium to terminate digestion after the cells became round; later, the cells were transferred into a sterile Eppendorf (EP) tube and centrifuged at $1000 \mathrm{r} / \mathrm{min}$ for $5 \mathrm{~min}$; after the original culture medium was discarded, the cells were added with fresh culture medium for resuspension, followed by subsequent experiment. SW480 cells in logarithmic growth phase were inoculated onto a 96-well plate, with $1 \times 10^{5}$ cells per well. After overnight culture, the cells were treated with different concentrations $(0,30$, 60 and $120 \mu \mathrm{M})$ of sulforaphane, cisplatin $(3 \mu \mathrm{M})$, or cisplatin+sulforaphane $(30,60$ and $120 \mu \mathrm{M})$ for $24 \mathrm{~h}$. Later, the original culture medium was removed and 20 $\mu \mathrm{l}$ of CCK-8 reagent was added into each well. Then, the cells were further cultured in a constant temperature incubator for $2 \mathrm{~h}$. Subsequently, the cell Absorbance (A) at $450 \mathrm{~nm}$ was measured using a microplate reader and the relative survival rate of cells (\%) was calculated as: $\mathrm{A}_{\text {experimental group }} / \mathrm{A}_{\text {control group }} \times 100 \%$. SW480 cells in logarithmic growth phase were inoculated onto a 6-well plate, with $2 \times 10^{6}$ cells per well. After $24 \mathrm{~h}$ of culture, the cells were treated with different concentrations $(0,30,60$ and $120 \mu \mathrm{M})$ of sulforaphane, cisplatin (3 $\mu \mathrm{M})$, or cisplatin $+60 \mu \mathrm{M}$ sulforaphane for $24 \mathrm{~h}$, followed by digestion and centrifugation. Then the cells were collected, washed twice with PBS, centrifuged and then added with $1 \times$ binding buffer to prepare cell suspension. Thereafter, the cell suspension was placed into a flow tube, added with Annexin V-FITC ( $5 \mu \mathrm{l})$ and Propidium Iodide (PI) $(5 \mu \mathrm{l})$. After mixing evenly, the cells were incubated in dark at room temperature for
15 min and then added with $1 \times$ binding buffer and fully mixed. Later, apoptosis was detected using a flow cytometer. The drug intervention was conducted as mentioned above. After the cells were collected and fully lysed on ice for $30 \mathrm{~min}$, the concentration of total protein was determined by BCA method. Next, $50 \mu \mathrm{g}$ of the sample was loaded in each well for gel electrophoresis (constant voltage of $70 \mathrm{~V}, 3 \mathrm{~h}$ ). After the proteins were completely separated, they were transferred onto a membrane (constant current of $275 \mathrm{~mA}, 70 \mathrm{~min}$ ). Later, the protein bands were blocked with $5 \%$ skimmed milk for $1 \mathrm{~h}$, incubated with primary antibodies (survivin, caspase-3, COX-2, Akt, p-Akt, GSK3 $\beta$, p-GSK3 $\beta$ and $\beta$-actin, dilution ratio of $1: 1000)$ at $4^{\circ}$ overnight and then the membrane was washed 3 times with TrisBuffered Saline-Tween 20 (TBST), followed by incubation with second antibodies (dilution ratio of 1:3000) at room temperature for $90 \mathrm{~min}$ and washing with TBST for 3 times. After ECL development and exposure in a dark room, the gray-scale values of the proteins were analyzed using the ImageJ software. Statistical Package for the Social Sciences (SPSS) 26.0 software was utilized for statistical analysis. The measurement data in line with normal distribution were expressed as mean \pm standard deviation. After variance analysis, Least Significant Difference (LSD)-t test was performed for comparisons between groups. $p<0.05$ indicated that a difference was statistically significant. No significant difference was found in the survival rate of SW480 cells between the low-dose $(30 \mu \mathrm{M})$ sulforaphane group and the control group $(\mathrm{p}>0.05)$. However, the survival rate in the other sulforaphane groups, cisplatin group and cisplatin+sulforaphane groups was significantly lower than that in control group $(\mathrm{p}<0.05)$. In addition, the survival rate in cisplatin+sulforaphane group was significantly lower than that in cisplatin group $(\mathrm{p}<0.05)$ and sulforaphane groups with the same dose of sulforaphane $(p<0.05)$ (fig. 1). The apoptosis rate of SW480 cells in control group, low-, medium- and high-dose sulforaphane groups, cisplatin group and cisplatin+medium-dose sulforaphane group were $(0.78 \% \pm 0.11 \%)$, $(1.23 \% \pm 0.11 \%),(2.78 \% \pm 0.12 \%),(3.89 \% \pm 0.12 \%)$, $(5.54 \% \pm 0.43 \%)$ and $(7.89 \% \pm 0.45 \%)$, respectively. It can be seen that the apoptosis rate of SW480 cells was significantly higher in sulforaphane groups (with different doses of sulforaphane), cisplatin group and cisplatin + medium-dose sulforaphane group than that in control group $(p<0.05)$ and it rose with the increase of sulforaphane concentration. In addition, the early apoptosis rate in cisplatin+medium-dose sulforaphane 


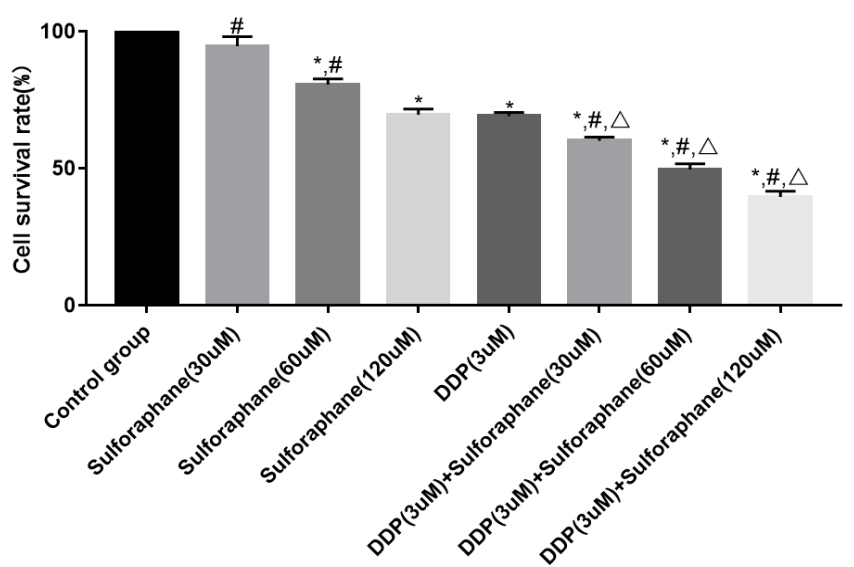

Fig. 1: Effect of sulforaphane on survival rate of $\mathrm{SW480}$ cells determined by $\mathrm{CCK}-8$ assay, ${ }^{*} \mathbf{p}<0.05$ vs. control group; ${ }^{\#} \mathbf{p}<0.05$ vs. cisplatin group; ${ }^{\wedge} \mathbf{p}<0.05$ : cisplatin+sulforaphane group $v s$. sulforaphane groups with the same dose of sulforaphane

group was significantly higher than that in sulforaphane groups (with different doses of sulforaphane) $(\mathrm{p}<0.05)$ (fig. 2). The expression level of survivin was significantly lower, while the expression level of caspase-3 was significantly higher in sulforaphane groups, cisplatin group and cisplatin+sulforaphane groups than that in control group $(\mathrm{p}<0.05)$. With the increase of sulforaphane dose, the expression level of survivin declined significantly, while the expression level of caspase-3 rose significantly, showing statistically significant differences among groups $(p<0.05)$. Moreover, the expression level of survivin was significantly lower, while the expression level of caspase-3 was significantly higher in cisplatin group than that in sulforaphane groups $(p<0.05)$. The expression level of survivin in cisplatin+sulforaphane groups was significantly higher than that in sulforaphane groups and cisplatin group $(p<0.05)$ (fig. 3$)$. The expression levels of COX-2, p-Akt and p-GSK3 $\beta$ in sulforaphane groups, cisplatin group and cisplatin+sulforaphane groups were significantly lower than those in control group $(\mathrm{p}<0.05)$. With the increase of sulforaphane dose, the expression levels of COX-2, p-Akt and p-GSK3 $\beta$ were declined significantly, showing statistically significant differences among groups $(p<0.05)$. In addition, the expression levels of COX-2, p-Akt and p-GSK3 $\beta$ were significantly lower in cisplatin group than those in sulforaphane group $(\mathrm{p}<0.05)$ and significantly lower in cisplatin+sulforaphane groups than those in sulforaphane groups and cisplatin group $(p<0.05)$ (fig. 4). Sulforaphane, derived from cruciferous plants, not only has strong anti-tumor activity ${ }^{[7,8]}$, but also shows good pharmacological effects on digestive diseases ${ }^{[9,10]}$. However, whether sulforaphane has potential value for adjuvant treatment of colon cancer needs further investigation. In this study, therefore, human colon cancer SW480 cells were adopted the in vitro research model and the effects of sulforaphane on the proliferation and apoptosis of SW480 cells were observed, whose mechanism was preliminarily discussed. Since the pathogenesis of tumor is not only associated with abnormal proliferation and differentiation of cancer cells, but also with inhibition of apoptosis, blocking the proliferation and inducing

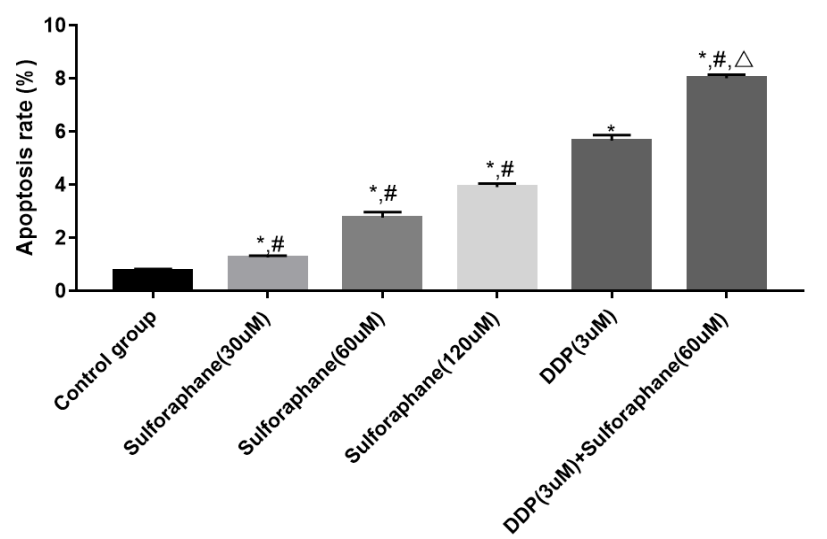

Fig. 2: Effect of sulforaphane on apoptosis rate of $\mathrm{SW480}$ cells, ${ }^{*} \mathbf{p}<0.05$ vs. control group; ${ }^{\#} \mathbf{p}<0.05$ vs. cisplatin group; ${ }^{\Delta} \mathbf{p}<0.05$ : cisplatin+sulforaphane group $v$ s. sulforaphane groups with the same dose of sulforaphane

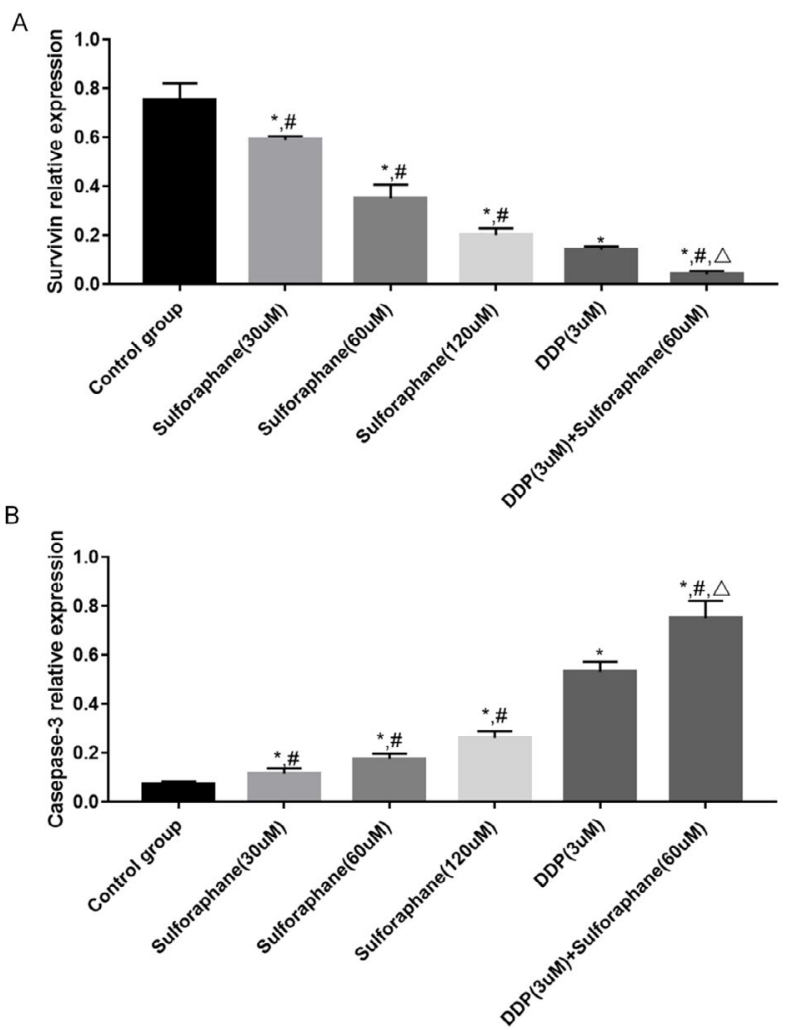

Fig. 3: Effect of sulforaphane on protein expressions of (A) Survivin and (B) Caspase-3 in SW480 cells, ${ }^{*} p<0.05$ vs. control group; ${ }^{\#} \mathbf{p}<0.05$ vs. cisplatin group; ${ }^{\Delta} \mathbf{p}<0.05$ : cisplatin+sulforaphane group $v s$. sulforaphane groups with the same dose of sulforaphane 


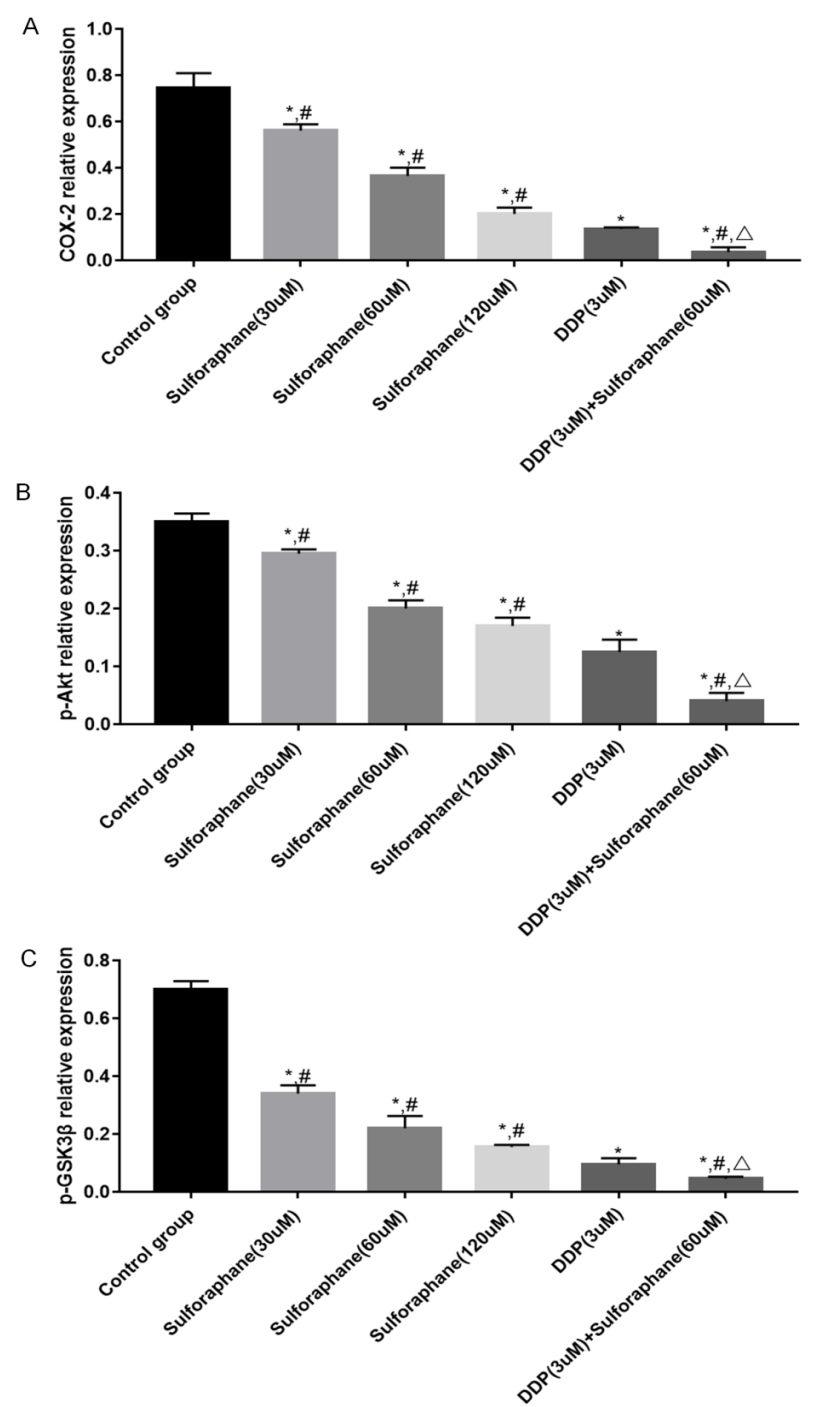

Fig. 4: Effect of sulforaphane on expression levels of (A) COX-2; (B) p-Akt and (C) p-GSK3及 in SW480 cells, *p<0.05 vs. control group; ${ }^{\#} \mathbf{p}<0.05$ vs. cisplatin group; ${ }^{\Delta} \mathbf{p}<0.05$ : cisplatin+sulforaphane group $v s$. sulforaphane groups with the same dose of sulforaphane

apoptosis of tumor cells may be the major means to prevent and treat cancer. Previous studies have proved that ${ }^{[11,12]}$ sulforaphane can exert an anti-tumor effect through inducing apoptosis of cancer cells, suppressing DNA synthesis in the cells and boosting cell immunity. Apoptosis is a gene-regulated autonomous programmed cell death. Such a biological effect can be modulated by the activation of various genes and the apoptosis pathways include death receptor pathway and mitochondrial pathway, both of which can lead to the activation of the effector caspase-3, resulting in endonuclease activation and thereby inducing apoptosis $^{[13-15]}$. Caspase is the major protein that regulates apoptosis, while survivin is a protein which inhibits apoptosis mainly through suppressing the activities of caspase- 3 and caspase- 7 and the expression levels of the above proteins are closely associated with the proliferation and apoptosis of human colorectal cancer cells ${ }^{[16,17]}$. In this study, the proliferation and apoptosis of SW480 cells were taken as the starting point to further investigate the molecular mechanism of sulforaphane. The results of CCK-8 assay demonstrated that sulforaphane suppressed the proliferation of SW480 cells in a concentration-dependent manner. Nevertheless, the inhibitory effect of different doses of sulforaphane on the growth of SW480 cells was less obvious than that of cisplatin alone and cisplatin + sulforaphane and the combination of cisplatin and sulforaphane showed a synergistic effect. Apoptosis detection results revealed that different doses of sulforaphane, cisplatin alone and cisplatin+sulforaphane could induce early apoptosis of SW480 cells and the early apoptosis rate was significantly positively correlated with the dose of sulforaphane. In addition, the apoptosis rate in cisplatin+sulforaphane groups was higher than that in sulforaphane groups and cisplatin group and the combination of cisplatin+sulforaphane had a synergistic effect. The results of Western blotting manifested that sulforaphane, cisplatin and the combination of cisplatin and sulforaphane could dramatically reduce the protein expression of survivin and induce the protein expression of caspase-3. COX-2 is highly expressed in human colorectal cancer cells. Early studies have proved that COX-2 can induce phosphorylation of Akt, thus promoting phosphorylation of GSK $3 \beta$ and the activation of this signal transduction can modulate biological processes such as cell proliferation, adhesion, differentiation and apoptosis $^{[18-20]}$. In this study, it was found that different doses of sulforaphane, cisplatin and cisplatin+sulforaphane could remarkably repress the expressions of COX-2, p-Akt and p-GSK3 $\beta$, which were positively correlated with the dose of sulforaphane. Moreover, the inhibitory effect of cisplatin+sulforaphane was more obvious than that of sulforaphane alone and cisplatin alone and the combination of cisplatin+sulforaphane displayed a synergistic effect. In summary, sulforaphane shows anti-cancer activity through inducing apoptosis and suppressing proliferation of colorectal cancer cells. Such a biological effect may be related to the suppression of the COX-2/ Akt/GSK3 $\beta$ signal transduction, up-regulation of the protein expression of caspase- 3 and down-regulation of the protein expression of survivin.

\section{Authors' contributions:}

Yongjun $\mathrm{Zhu}$ and Haijun $\mathrm{Hu}$ have contributed equally to this work. 


\section{Conflict of interests:}

The authors declared no conflicts of interest.

\section{REFERENCES}

1. Thangapandiyan S, Ramesh M, Hema T, Miltonprabu S, Uddin MS, Nandhini V, et al. Sulforaphane potentially ameliorates arsenic induced hepatotoxicity in albino wistar rats: Implication of PI3K/Akt/Nrf2 signaling pathway. Cell Physiol Biochem 2019;52(5):1203-22.

2. Iida Y, Okamoto-Katsuyama M, Maruoka S, Mizumura K, Shimizu T, Shikano S, et al. Effective ferroptotic smallcell lung cancer cell death from SLC7A11 inhibition by sulforaphane. Oncol Lett 2021;21(1):1.

3. Chen Y, Wang MH, Zhu JY, Xie CF, Li XT, Wu JS, et al. TAp63 $\alpha$ targeting of Lgr5 mediates colorectal cancer stem cell properties and sulforaphane inhibition. Oncogenesis 2020;9(10):1.

4. Zhang YC, Zhao H, Chen C, Ali MA. COX-2 gene rs689466 polymorphism is associated with increased risk of colorectal cancer among Caucasians: A meta-analysis. World J Surg Oncol 2020;18(1):1-9.

5. Zhou C, Cui F, Li J, Wang D, Wei Y, Wu Y, et al. MiR650 represses high-risk non-metastatic colorectal cancer progression via inhibition of AKT2/GSK3 $\beta /$ E-cadherin pathway. Oncotarget 2017;8(30):49534-47.

6. Chen WF, Gao WD, Li QL, Zhou PH, Xu MD, Yao LQ. SLIT2 inhibits cell migration in colorectal cancer through the AKT-GSK3 $\beta$ signaling pathway. Int $\mathrm{J}$ Colorectal Dis 2013;28(7):933-40.

7. Hossain S, Liu Z. DICKKOPF-1 (DKK-1) Gene associations in human cancers by vitamin D and sulforaphane. J Cancer Sci Clin Ther 2020;4(3):237-44.

8. Gu HF, Mao XY, Du M. Metabolism, absorption and anticancer effects of sulforaphane: An update. Crit Rev Food Sci Nutr 2020:1-7.

9. Hao WU, Xiao-sheng LU, Jie-qiang LY. Biological effects of sulforaphane and its role in female reproductive system. Int $\mathbf{J}$ Gynaecol Obstet 2019;46(2):216.

10. Pastorek M, SIMkO V, Takacova M, Barathova M, Bartosova M, Hunakova L, et al. Sulforaphane reduces molecular response to hypoxia in ovarian tumor cells independently of their resistance to chemotherapy. Int J Oncol 2015;47(1):5160.

11. Kaminski BM, Steinhilber D, Stein JM, Ulrich S. Phytochemicals resveratrol and sulforaphane as potential agents for enhancing the anti-tumor activities of conventional cancer therapies. Curr Pharm Biotechnol 2012;13(1):137-46.

12. Chen MJ, Tang WY, Hsu CW, Tsai YT, Wu JF, Lin CW, et al. Apoptosis induction in primary human colorectal cancer cell lines and retarded tumor growth in SCID mice by sulforaphane. Evid Based Complement Alternat Med 2012;2012.

13. Hirai T, Konishi Y, Mizuno S, Rui Z, Sun Y, Nishiwaki K. Differential effects of sevoflurane on the growth and apoptosis of human cancer cell lines. J Anesth 2020;34(1):47-57.

14. Li N, Zhang Y, Fan Y. The effects of silencing of POLE2 gene expression on proliferation of non-small cell lung cancer cells. J Mod Oncol 2019;27(1):35-40.

15. Jin B, Huang R, Liu Q. Effects of puerarin on apoptosis of prostate cancer cells through TGF- $\beta$ /Smads signaling pathway. Xiandai Zhongliu Yixue 2020;28(1):17-22.

16. Pisarska M, Torbicz G, Gajewska N, Rubinkiewicz M, Wierdak M, Major P, et al. Compliance with the ERAS protocol and 3-year survival after laparoscopic surgery for non-metastatic colorectal cancer. World J Surg 2019;43(10):2552-60.

17. Volpato $\mathrm{M}$, Ingram N, Perry SL, Spencer J, Race AD, Marshall C, et al. Cyclooxygenase activity mediates colorectal cancer cell resistance to the omega- 3 polyunsaturated fatty acid eicosapentaenoic acid. Cancer Chemother Pharmacol 2021;87(2):173-84.

18. Khalaf S, Toor SM, Murshed K, Kurer MA, Ahmed AA, Nada MA, et al. Differential expression of TIM-3 in circulation and tumor microenvironment of colorectal cancer patients. Clin Immunol 2020;215:108429.

19. Zhi H, Lian J. LncRNA BDNF-AS suppresses colorectal cancer cell proliferation and migration by epigenetically repressing GSK-3 $\beta$ expression. Cell Biochem Funct 2019;37(5):340-7.

20. Wei W, Ma XD, Jiang GM, Shi B, Zhong W, Sun CL, et al. The AKT/GSK3 $\beta$-mediated slug expression contributes to oxaliplatin resistance in colorectal cancer via upregulation of ERCC1. Oncol Res 2020;28(4):423-38. 